\title{
Fasciola hepatica extract suppresses fibroblast-like synoviocytes in vitro and alleviates experimental arthritis
}

\section{Suelen Pizzolatto Dalmolin}

Hospital de Clínicas de Porto Alegre

\section{Renata Ternus Pedó}

Hospital de Clínicas de Porto Alegre

Thales Hein Rosa

Hospital de Clínicas de Porto Alegre

Jordana Miranda Souza Silva

Hospital de Clínicas de Porto Alegre

Mirian Farinon

Hospital de Clínicas de Porto Alegre

Maria Luísa Gasparini

Hospital de Clínicas de Porto Alegre

\section{Eduardo Cremonese Filippi Chiela}

Universidade Federal do Rio Grande do Sul

\section{Ana Helena Paz}

Universidade Federal do Rio Grande do Sul

Martín Pablo Cancela Sehabiague

Universidade Federal do Rio Grande do Sul

Henrique Bunselmeyer Ferreira

Universidade Federal do Rio Grande do Sul

\section{Rafaela Cavalheiro Espírito Santo}

Hospital de Clínicas de Porto Alegre

\section{Fabiany Gonçalves}

Erasmus Medical Center

Ricardo Machado Xavier ( $\square$ rmxavier@hcpa.edu.br)

Hospital de Clínicas de Porto Alegre

\section{Research Article}

Keywords: Rheumatoid arthritis, fibroblast-like synoviocytes, antigen-induced arthritis, collagen-induced arthritis, Fasciola hepatica 
Posted Date: February 17th, 2022

DOI: https://doi.org/10.21203/rs.3.rs-1327551/v1

License: (c) (i) This work is licensed under a Creative Commons Attribution 4.0 International License. Read Full License 


\section{Abstract}

Rheumatoid arthritis (RA) is a chronic inflammatory disease characterized by synovial inflammation, fibroblast-like synoviocytes (FLS) activation and joint destruction. Fasciola hepatica is a platyhelminth that releases excretory-secretory immunomodulatory products capable of suppressing the Th1 immune response and the production of inflammatory cytokines. Despite the effectiveness of available treatments for inducing and maintaining disease remission, there is no cure for RA. Current options are not successful in all patients and may cause side effects. Thus, new therapeutic alternatives are needed for RA. We evaluated the therapeutic potential of $F$. hepatica extract on FLS from RA patients and in antigeninduced (AIA) and collagen-induced (CIA) arthritis models. FLS were isolated from synovial fluid of RA patients, cultured, and exposed to $F$. hepatica extract $(60,80$, and $100 \mu \mathrm{g} / \mathrm{ml})$ for different time points. In vitro assays showed that extract decreased FLS cell viability, adherence, migratory potential, and cell invasiveness. For in vivo experiments, AIA and CIA animals received a $200 \mu \mathrm{g} /$ dose of $F$. hepatica extract daily. The extract reduced leukocyte migration and nociception in AIA and delayed clinical onset of $\mathrm{CIA}$, while preventing body weight loss. Taken together, our results point out a potential immunomodulatory effect of the $F$. hepatica extract in RA models.

\section{Introduction}

Rheumatoid arthritis (RA) is a chronic inflammatory disease that occurs in $0.5-1.0 \%$ of the adult population worldwide ${ }^{1}$ and is characterized by synovial hyperplasia with invasion and destruction of cartilage and bone ${ }^{2}$. Synovial joints cover the articular space and produces the synovial fluid for the joints ${ }^{2}$. The synovial membrane mainly consists of fibroblast-like synoviocytes (FLS) and during the progression of RA, a subpopulation of these cells acquire a modified phenotype that is aggressive and locally invasive ${ }^{3}$. In addition to contributing to the inflammatory environment, the ability to invade and promote cartilage destruction stands out as a main characteristic of FLS in RA. Thus, these cells are considered one of the main tissue components in the initiation and perpetuation of RA, as well as in the process of joint destruction 2,4 .

Although the available treatments are relatively effective for inducing and maintaining disease remission, there is no known cure for RA ${ }^{5,6}$. In addition to the steroid and non-steroid anti-inflammatory drug options, disease-modifying antirheumatic drugs (DMARDs) can alter the course of RA ${ }^{7}$. The DMARDs can be conventional and targeted synthetic DMARDs, as well as biologic DMARDs. However, these options have not been successful in all patients and could cause a number of side effects, some of which involve significant health care costs ${ }^{8}$. Thus, new therapeutic alternatives are needed for RA.

In this context, parasite-secreted proteins with the potential to modulate the immune system could be explored as a therapeutic approach for RA and other autoinflammatory diseases ${ }^{9}$. In fact, parasitemediated regulatory effects on the immune system have been reported in immune mediated diseases, such as RA ${ }^{10,11}$, multiple sclerosis ${ }^{12}$, inflammatory bowel diseases, such as Crohn's disease ${ }^{13,14}$ and 
ulcerative colitis ${ }^{13}$, as well as in celiac disease ${ }^{11}$, type 1 diabetes ${ }^{15}$, and allergies ${ }^{16}$. Among the helminths with immunomodulatory characteristics, Fasciola hepatica has been studied for this purpose. The tegument covering this parasite has a layer rich in secretory inclusions that is externally limited by a dense glycocalyx plasma membrane ${ }^{17,18}$. F. hepatica tegument is highly glycosylated, with the presence of oligosaccharide motifs and glycoproteins, which have immunomodulatory properties ${ }^{19}$. The interaction with the host immune cells occurs through constant tegument shedding 20,21.

To the best of our knowledge, no studies have assessed the effect of $F$. hepatica extract on FLS derived from RA patient or animal models of arthritis. In this study, we evaluated the therapeutic potential of $F$. hepatica extract in FLS from patients with $\mathrm{RA}^{9}$ and in acute and chronic mice models of arthritis.

\section{Materials And Methods}

\section{Study design and patients}

This cross-sectional study was performed at a tertiary public hospital in Rio Grande do Sul, Brazil (Hospital de Clínicas de Porto Alegre, HCPA). Patients diagnosed with RA according to the American College of Rheumatology/American College of Rheumatology/European League Against Rheumatism (ACR/EULAR) criteria ${ }^{45}$, with active disease and with indication of knee arthrocentesis by the attending rheumatologist were included in study. Patients with positive infection serology tests (HIV, HBV, HCV, syphilis, tuberculosis) and indication of arthrocentesis on other joints were excluded. The disease activity score (DAS) was calculated with DAS28-erythrocyte sedimentation rate. The study was approved by the HCPA Hospital Research Ethics Committee (CEP) and written informed consent was obtained from each patient, complying with all methods in accordance with CEP guidelines and regulations.

\section{Collection procedure of synovial liquid}

The arthrocentesis procedure was performed by a rheumatologist. Briefly, local anesthetic was used in the area of the joint and a needle with a syringe attached is inserted within the joint (joint injection) and joint fluid is drawn back under suction (aspirated) into the syringe. The synovial liquid was placed in a 15 $\mathrm{ml}$ falcon tube and stored in ice until processing.

\section{Isolation and Culture of FLS cells}

The synovial fluid of RA patients was processed for the isolation of FLS. The liquid was centrifuged at 2000 x g for 5 min, resuspended in Dulbecco's Modified Eagle's Medium - High glucose (DMEM-HG, Gibco by Life Technologies, USA) supplemented with $15 \%$ fetal bovine serum (FBS, Gibco), 200 mM L-glutamine (Gibco), 100 units/mL penicillin (Gibco), $100 \mathrm{mg} / \mathrm{mL}$ streptomycin (P/S) (Gibco), $50 \mathrm{mg} / \mathrm{mL}$ of gentamicin and transferred to a 6-well plate for culture (Thermo Fisher Scientific, USA). Cells were allowed to attach during three days before replacing the growth medium. The cultures were kept at $37^{\circ} \mathrm{C}$ in a $5 \% \mathrm{CO}_{2}$ atmosphere, and the medium was replaced every 3 days. The initial culture was monitored until $70-80 \%$ of 


\section{confluence and then cells were detached with trypsin-EDTA (Gibco) and transferred to a culture flask for the tests. FLS between 5 and 11 passages were used for experiments.}

\section{Preparation of $F$. hepatica extract}

Mature $F$. hepatica was extracted from the bile ducts of bovine livers obtained at local abattoirs and extensively washed at $37^{\circ} \mathrm{C}$ in $10 \mathrm{mM}$ phosphate-buffered saline (PBS) $\mathrm{pH}$ 7.3. Only clean and viable parasites were used for further downstream procedure. Adult liver flukes $F$. hepatica extract was obtained by homogenization ( $1 \mathrm{~g}$ of adult parasite/10 mL PBS) using a glass tissue grinder and then sonicated in ice bath five times with $60 \mathrm{~s}$ bursts at $20 \%$ power followed by $30 \mathrm{~s}$ pauses. After centrifugation at 20000 $\mathrm{g}$ for 30 min at $4{ }^{\circ} \mathrm{C}$, supernatant was stored at $-80^{\circ} \mathrm{C}$ until use ${ }^{46}$. Protein concentration was determined by using Qubit fluorimetric assay (Invitrogen).

\section{Morphology and Characterization of FLS}

Morphological analysis of FLS was performed by phalloidin staining of actin filaments (Sigma-Aldrich by Merck, St. Louis, EUA). FLS $\left(0.7 \times 10^{4}\right.$ cells $\left./ \mathrm{ml}\right)$ were cultured over microscopy coverslips $(18 \times 18 \mathrm{~mm})$ and placed into 12-well culture plates. After $48 \mathrm{~h}$, adhered cells were fixed for $15 \mathrm{~min}$ with $4 \%$ paraformaldehyde at RT in dark. Then, the cells were washed three times with PBS and blocked with $3 \%$ bovine serum albumin (BSA) (Sigma-Aldrich) for $1 \mathrm{~h}$ at RT in dark. Staining was performed with $50 \mathrm{nM}$ Alexa 488 conjugated phalloidin (Sigma-Aldrich) for $1 \mathrm{~h}$ at RT in dark. Nuclei were stained with (300 nM) of 4',6-diamidino-2-phenylindole solution (DAPI; Sigma-Aldrich, Missouri, USA). Finally, cells were photographed in a fluorescence microscope with appropriate filters (Olympus IX-71, Olympus Corporation, Tokyo, Japan). For phenotyping characterization, FLS were used in passage 5. Cells were centrifuged (2000 $\mathrm{x} \mathrm{g}$ for $5 \mathrm{~min}$ ), the supernatant was aspirated, and the antibodies were applied directly onto the pellet. After, the FLS were incubated with the following monoclonal antibodies: anti-Thy-1 (CD90), antiCD55 (both FLS positive) and anti-CD68 (macrophage positive) (BD Biosciences, New Jersey, EUA). After incubation for 15 min at dark and room temperature (RT), cells were washed with PBS, centrifuged, resuspended and analyzed by flow cytometry (Attune, Applied Biosystems, Life Technologies). Unstained cells were used as controls.

\section{Viability assay (MTT)}

FLS viability was determined by MTT [3-(4,5-dimethylthiazol-2-yl)-2,5-diphenyltetrazolium bromide] assay. The FLS were seeded in 96-well plates at a density of $5 \times 10^{3} \mathrm{cell} / \mathrm{ml}$ and exposed to different doses of $F$. hepatica extract $(60,80$, and $100 \mu \mathrm{g} / \mathrm{ml})$ for 24,48 , and $72 \mathrm{~h}$ at $37^{\circ} \mathrm{C}$ in $5 \% \mathrm{CO}_{2}$. Parallel controls were carried out with PBS (vehicle control) or untreated group (control). The extract were replaced every day. Then, the culture medium was replaced with MTT $(0.5 \mathrm{mg} / \mathrm{ml})$ and incubated for $4 \mathrm{~h}$. The supernatants were removed and dimethyl sulphoxide (DMSO, Sigma Aldrich) was added to dissolve the MTT formazan crystals. The absorbance of each well was measured at $570 \mathrm{~nm}$ and $690 \mathrm{~nm}$ (SpectraMax M3) spectrophotometer and background was discounted. Cell viability was expressed as percent of average of control (or vehicle control). Eight experiments were performed independently $(n=8)$. 


\section{Nuclear Morphometric Analysis (NMA)}

A nuclear morphometric analysis (NMA) was performed as described by Filippi-Chiela et al. (2012). FLS were seeded in 24-well plates at a density of $1 \times 10^{4}$ cells $/ \mathrm{ml}$. The following day, FLS were treated with $100 \mu \mathrm{g} / \mathrm{mL}$ F. hepatica extract for $48 \mathrm{~h}$. After $24 \mathrm{~h}$, cells were fixed with $4 \%$ paraformaldehyde and stained with $100 \mu \mathrm{L}(300 \mathrm{nM})$ of 4',6-diamidino-2-phenylindole solution (DAPI; Sigma-Aldrich, Missouri, USA). Then, cells were photographed in a fluorescence microscope with appropriate filters (Olympus IX-71, Olympus Corporation, Tokyo, Japan) and a total of 50 nuclei was obtained from random fields. The images were analyzed using Image Pro-Plus 6.0 software (Media Cybernetics, Maryland, USA), for the acquisition of the nuclear area and the following parameters of nuclear shape: roundness, aspect, radius ratio and area/box. These four variables of shape were grouped in an index, named the Nuclear Irregularity Index (NII). The Area versus NII plot classified each nuclei according to its morphometric. Three experiments were performed independently $(n=3)$.

\section{Apoptosis and necrosis assay (Annexin V/Propidium lodide [PI])}

FLS were plated in a 24-well plate at a density of $5 \times 10^{4}$ cells/ $\mathrm{ml}$ for apoptosis and cell death detection. FLS were treated with $100 \mu \mathrm{g} / \mathrm{mL}$ of $F$. hepatica extract or PBS for $48 \mathrm{~h}$. The test was performed by the Annexin V-fluorescein isothiocyanate (FITC)/Propidium lodide (PI) kit (Gibcol Thermo Fisher Scientific) according to the manufacturer's instructions. Briefly, FLS were washed and resuspended in Annexin $\mathrm{V}$ binding buffer, centrifuged at $1600 \mathrm{xg}$ for $5 \mathrm{~min}$, and labeled with Annexin V-FITC and PI. After $10 \mathrm{~min}$ at RT in dark, Annexin $\mathrm{V}$ binding buffer was added and centrifuged one more time. The resulting fluorescence was detected by flow cytometry (Attune, Applied Biosystems, Life Technologies). Two experiments were performed independently $(n=4)$.

\section{Adherence assay}

To evaluate the adherence potential of FLS, cells were seeded in 96-well plate at a density of $1 \times 10^{2}$ cells $/ \mathrm{ml}$ and exposed to $100 \mu \mathrm{g} / \mathrm{ml} F$. hepatica extract or PBS for $6 \mathrm{~h}$. After $6 \mathrm{~h}$, cells were counted immediately in an optical microscopy for adherence to polystyrene plate evaluation. Adherence was expressed by the number of adhered cells divided by the number of seeded cells. Four experiments were performed independently $(n=4)$.

\section{Wound healing assay}

The wound closure motility assay was performed by seeding $3.5 \times 10^{4} \mathrm{cells} / \mathrm{ml}$ in 24 -well plates overnight and by treating them with $100 \mu \mathrm{g} / \mathrm{mL}$ F. hepatica extract or PBS for $48 \mathrm{~h}$. A linear wound was created using a $200 \mu \mathrm{L}$ micropipette tip and then washed with medium to remove unattached cells. Light microscopy images were taken immediately 0 and $24 \mathrm{~h}$ after wounding and, at the end of the experiment, cells were fixed with $4 \%$ paraformaldehyde and stained with crystal violet. The distance of the wound was measured in "inch" by ImageJ software and was expressed as percent of the average of control. Four experiments were performed independently $(n=4)$. 


\section{Invasion assay}

To perform the invasion capacity analysis, FLS were treated with $100 \mu \mathrm{g} / \mathrm{mL}$ of $F$. hepatica or PBS for 48 $\mathrm{h}$ in T75 culture flasks. After, the FLS were seeded in matrigel inserts (collagen matrix) Corning ( 24 well plates, 8-mm pore diameter; Bedford, MA, USA) at a density of $1 \times 10^{5}$ cells/well. The test was performed according to the manufacturer's specifications. To measure cell invasion, FLS were seeded in medium free of FBS in the upper chamber. Medium supplemented with 15\% FBS was used as an attractant in the lower chamber. After $24 \mathrm{~h}$, cells that invaded through the matrix were stained with Crystal Violet, photographed by microscope, and analyzed in the ImageJ software. Two experiments were performed independently $(n=3)$.

\section{Enzyme-linked immunosorbent assay (ELISA)}

To evaluate the TNF-a expression of FLS, a density of $1 \times 10^{4}$ cells $/ \mathrm{ml}$ were seeded in a 24-well plate for ELISA assay. When cells reached the confluence of $80 \%$, the culture medium was suplemmented with 2 $\mu \mathrm{L} / \mathrm{mL}$ of interferon-gamma (INF- $\gamma$ - Thermo Fisher) for 3 days to induce an inflammatory condition in vitro. After that, FLS were treated with $100 \mu \mathrm{g} / \mathrm{mL}$ F.hepatica extract or PBS for $48 \mathrm{~h}$. Then, the medium was replaced and the supernatant was collected after $24 \mathrm{~h}$ and stored at $-80^{\circ} \mathrm{C}$ until use. The concentration of TNF-a in supernatants was measured by human TNF-a Elisa Max ${ }^{\mathrm{TM}}$ Standard Set (BioLegend, San Diego, CA, USA). The absorbance of each well was measured at $450 \mathrm{~nm}$ and $570 \mathrm{~nm}$ in SpectraMax M3 spectrophotometer. Data analyses were performed in My Assays software ("Four Parameter Logistic Curve" online data analysis tool, MyAssays Ltd, Sussex, UK) and they are representative at $\mathrm{pg} / \mathrm{mL}$. Three experiments were performed independently $(n=3)$.

\section{Animals}

Balb/C mice (male, 8-12 weeks, 20-25 g) were used for AIA model, while DBA/1 J mice (male, 8-12 weeks, 18-22 g) were used for CIA model. All animals were kept under a $12 \mathrm{~h}$ light-dark cycles, 40-60\% relative humidity, and temperature of $22 \pm 2{ }^{\circ} \mathrm{C}$ with free access to water and food. The study complies with the principles of the 3Rs: Replacement of animals by alternatives wherever possible, Reduction in number of animals used, and Refinement of experimental conditions and procedures to minimize the harm to animals. Moreover, all experimental procedures were performed following recommendations of the National Institute of Health Guide for Care and Use of Animals and with the approval by the Ethics Committee for the Use of Animals (CEUA) of the Hospital de Clínicas de Porto Alegre (project identification code: 17-0310, approval date: 26/07/2017), complying with all methods in accordance with CEUA guidelines and regulations. All animals were anesthetized with isoflurane (Abbott, Abbott Park, IL, USA) for arthritis induction and euthanasia and were monitored twice a week to assess any behavioral change and loss of quality of life, observing signals of inactivity, suffering, stress, unbearable pain and no food or water intake.

\section{Induction of AIA}


Fourteen Balb/c mice were sensitized on day 0 with a subcutaneous injection of $200 \mu \mathrm{l}$ of a solution containing an equal ratio of $500 \mu \mathrm{g}$ methylated bovine serum albumin (mBSA) (Sigma-Aldrich, Saint Louis, MO, USA) diluted in saline and complete Freund's adjuvant (CFA) (Sigma-Aldrich). On day 7 and day 14 mice were subjected to booster subcutaneous injections of mBSA with incomplete Freund's adjuvant (IFA) (Sigma-Aldrich). Arthritis was induced on day 21, with an intra-articular (ia) injection of 30 $\mu \mathrm{g}$ of mBSA into the left knee joint. The right knee joint was injected with saline $0.9 \%$ as a negative control of arthritis (sham group) ${ }^{47}$. Animals were randomly divided into two groups and then were treated with $F$. hepatica extract in the dosage of $200 \mu \mathrm{g}$ (extract group) or PBS (control group). Treatment was performed intraperitoneally in $100 \mu \mathrm{l} 24 \mathrm{~h}$ and 30 min prior to mBSA joint injection.

\section{Evaluation of joint nociception and leukocyte migration in AIA}

Nociception was measured at 0 h, 3 h, 6 h, and 24 h after mBSA injection using a digital analgesiometer (Insight Instruments, Ribeirão Preto, SP, Brazil). After environmental adaptation, a force was applied to the hind paws of mice and the equipment measured the intensity of the force when the paw was withdrawn, with results expressed as the flexion-elicited withdrawal threshold in grams. Leukocyte migration was evaluated $24 \mathrm{~h}$ after mBSA injection. After euthanasia, the knee cavities were washed three times with 5 $\mu \mathrm{l}$ of PBS-EDTA and then diluted in a final volume of $90 \mu \mathrm{l}$. The recovered liquid was mixed in Turk's solution (1:1) and the total number of leukocytes was counted using a Neubauer Chamber (HBG, GiessenLützellinden, Germany) under an optical microscope (Olympus, Tokyo, Japan).

\section{Induction of CIA}

Sixteen DBA/ $1 \mathrm{~J}$ mice were immunized with $70 \mu \mathrm{l}$ of an emulsion containing an equal volume of $2 \mathrm{mg} / \mathrm{ml}$ bovine collagen type II (CII) (Chondrex, Redmond, WA, USA) and CFA with $2 \mathrm{mg} / \mathrm{ml}$ of heatinactivated Mycobacterium tuberculosis (Strain H37 RA) (Difco Laboratories, Lawrence, KS, USA) by intradermal injection in the base of the tail on day 0 . After eighteen days, a booster injection was performed in another site of the tail with $70 \mu$ of an emulsion with CII and IFA ${ }^{38}$. Animals were randomized into two groups: arthritis-induced animals treated with PBS (control group) and arthritisinduced animals treated with $F$. hepatica extract at the dosage of $200 \mu \mathrm{g}$ (extract group). Animals were treated once a day by intraperitoneal injection and treatment started after booster lasting for 28 days (46 days of experimentation).

\section{Clinical arthritis scoring of CIA}

After booster injection, animals were monitored every other day for clinical signs of arthritis using the following score: 0 -normal, 1-mild swelling and erythema, 2-moderate swelling and erythema, 3-severe swelling and erythema extending from the ankle to metatarsal joints and 4-severe erythema and swelling with loss of function. The total score of the animal is presented as the sum of the score in each paw (range: $0-16$ ) and the score of hind paws of the animals is presented as the mean of both hind paws (range: $0-4)^{38}$. 


\section{Statistical analyses}

Data are presented as mean \pm standard deviation (SD). Groups were compared by one-way or two-way analysis of variance (ANOVA) and by Student's t-test using GraphPad Prism 6.0. Statistical differences were considered significant with a $p$ value $<0.05$.

\section{Results}

\section{Clinical and serological characteristics of RA patients}

Synovial fluid was collected from a total of thirty-two patients with established RA. Of the 32 FLS isolates, 12 cellular cultures grew appropriately in vitro and were used in this study. These were derived from 7 females and 5 males, mean age 65 years, mean duration of disease 15 years. Four of the patients were positive for anti-cyclic citrullinated peptides (anti-CCP) antibodies and presented a disease activity score (DAS-28) of 3,61 (IQR: 3,3-4.7) (2 high, 9 moderate, and 1 low disease activity). Most of the patients (33.3\%) received treatment with DMARD and concurrent glucocorticoids. The baseline demographic, clinical, and laboratory parameters can be found in Supplementary Table S1 online.

Morphology and Characterization of FLS

To evaluate the morphology of FLS, cells collected from RA patients were cultured and stained with Alexa 488 conjugated phalloidin and analyzed by fluorescence microscopy. The FLS cells showed the classic FLS elongated phenotype (Figure 1A). To determine the purity of FLS cultures, the cells were tested with flow-cytometric analysis using FITC- Mouse Anti-Human CD90, PE- Mouse Anti-Human CD55 (both FLS positive) and FITC- Mouse Anti-Human CD68 (macrophage positive) monoclonal antibodies (Figure 1B). Most cells expressed the surface markers for FLS, such as CD55 (87 $\pm 4.5 \%)$ and CD90 $(92 \pm 6.0 \%)$ and they were negative for CD68 (1\% \pm 1.1$)$ (Figure 1C).

\section{F. hepatica extract decreases the cellular viability of FLS}

To analyze FLS viability after treatment with $F$. hepatica extract, the FLS were exposed to different doses $(0,60,80$, and $100 \mu \mathrm{g} / \mathrm{mL})$ and time points $(24,48$, and $72 \mathrm{~h})$. The $F$. hepatica extract decreased the cell viability of FLS at concentration of $100 \mu \mathrm{g} / \mathrm{ml}$ after $48 \mathrm{~h}(83 . \% \pm 5.0$ extract $v s 100 . \% \pm 0.0$ control; $p<$ $0.05)$ and at concentrations of $80 \mu \mathrm{g} / \mathrm{ml}(88 . \% \pm 3.0$ extract $v s 100 . \% \pm 0.0$ control; $p<0.05)$, and 100 $\mu \mathrm{g} / \mathrm{ml}(89 . \%$ extract \pm 3.8 extract $v s 100 . \% \pm 0.0$ control; $p<0.05)$ after $72 \mathrm{~h}$, when compared with control group without treatment $(n=8)$. There was no significant difference in cell viability at $60 \mu \mathrm{g} / \mathrm{ml}$ and between the time of $48 \mathrm{~h}$ and $72 \mathrm{~h}$ at $100 \mu \mathrm{g} / \mathrm{ml}$. Based on these results, the $100 \mu \mathrm{g} / \mathrm{ml}$ dose and 48 $\mathrm{h}$ of exposure were chosen as the treatment protocol for the subsequent in vitro analyses (Figure 2).

\section{F. hepatica extract did not alter nuclear morphometrics and did not cause cell death or senescence of FLS}

Nuclear size and shape can indicate several cell fates, including mitosis, apoptosis, senescence, or mitotic catastrophe. To evaluate the nuclear morphometrics, the cells were treated with $F$. hepatica extract 
for $48 \mathrm{~h}$, followed by DAPI staining and analysis in Image Pro Plus 6.0. Treatment did not alter the nuclear morphometrics of FLS in area, roundness, aspect, radius ratio, or area box (Figure 3A/ Figure 3B) $(n=3)$. To assess apoptosis and necrosis, FLS were labeled with annexin and propidium iodide and were analyzed by flow cytometry (Figure $3 C$ ). The $F$. hepatica extract did not induce apoptosis or cell death (Figure 3D) $(n=4)$.

\section{F. hepatica extract decreased FLS adherence efficiency, invasion, and migratory potential}

To analyze FLS adherence efficiency, the cells were seeded in a culture plate, immediately treated with $F$. hepatica extract and analyzed after $6 \mathrm{~h}$ by optical microscopy. F. hepatica extract decreased the FLS capacity to adhere in $20 \%$ compared with the control group ( 92.0 cells \pm 5.8 extract vs 116.3 cells \pm 7.9 control; $p<0.05)$; $(\mathrm{n}=4)$ (Figure 4A). To evaluate the invasion potential of FLS, the cells were treated with F. hepatica extract for $48 \mathrm{~h}$ and then cultured in matrigel inserts for $24 \mathrm{~h}$. Treatment with extract reduced FLS invasion potential through the matrigel compared with the control group $(76.0 \% \pm 8.4$ extract $v s$ $100.0 \%$ control; $p<0.01)(n=3)$ (Figure 4B). To determine the FLS migratory potential, FLS were treated with the $F$. hepatica extract for $48 \mathrm{~h}$ and then a linear wound was created in the well. Light microscopy images were taken immediately 0 and $24 \mathrm{~h}$ after wounding and images were analyzed by Image $\mathrm{J}$. The distance of the wound was measured in "inch" and expressed as percent of the average of control. F.hepatica extract reduced migratory potential of FLS compared with the control group $(69.5 \% \pm 17.6$ extract vs $100.0 \%$ control; $p<0.05)(n=4)(F i g u r e 4 C)$. TNF, an important cytokine involved in the RA, were analyzed on FLS supernatants after stimulation with INF- $\gamma$ for $72 \mathrm{~h}$ to induce an inflammatory condition. After treatment, the cell supernatant was placed in an ELISA plate for TNF quantification. Although $F$. hepatica treatment tended to decrease TNF release compared to the control group, this difference was not statistically significant $(8.55 \pm 0.51 \mathrm{pg} / \mathrm{mL}$ control vs $7.01 \pm 0.52 \mathrm{pg} / \mathrm{mL}$ extract).

\section{F. hepatica extract reduces nociception and leukocyte chemotaxis in acute antigen-induced arthritis}

To analyze the in vivo effects of $F$. hepatica extract in mouse models of RA, we first evaluated the analgesic potential of $F$. hepatica extract in acute methylated bovine serum albumin (mBSA) antigeninduced arthritis (AIA) (Figure 5A), considering increased pain in response to the mBSA injection and inflammatory process. Nociception in control group mice increased $3 \mathrm{~h}$ after the mBSA injection, peaking at $24 \mathrm{~h}$. F. hepatica extract treatment reduced nociception, with treated mice enduring 1.7 times more nociceptive stimulus than control $(6.37 \mathrm{~g} \pm 0.99$ extract vs $3.81 \mathrm{~g} \pm 1.44$ control; $\mathrm{p}<0.001) 24 \mathrm{~h}$ after the mBSA injection (Figure 5B).

Leukocyte migration to the knee joint is a consequence of the inflammatory response to mBSA antigen, being an important marker of local inflammatory activity ${ }^{22}$. Therefore, this parameter is a useful predictor of anti-inflammatory effect. As expected, mBSA injection provoked massive recruitment of leukocytes to the knee joint cavity of the control group, compared to saline injection in the contralateral joint of the same mice (sham group). Treatment with $F$. hepatica extract inhibited leukocyte chemotaxis 
by $56 \%\left(40 \times 10^{4}\right.$ leukocytes/knee \pm 19.00$)$ compared to control $\left(90.90 \times 10^{4}\right.$ leukocytes/knee \pm 12.90$)$ $(p<0.01)$ (Figure 5C).

Treatment with F. hepatica extract delayed the beginning of clinical signs and prevented body weight loss in collagen-induced arthritis

After confirming the efficacy of $F$. hepatica extract treatment in the initial process of arthritis development, we analyzed its effects in the chronic phase of the disease by performing collagen-induced arthritis (CIA) model (Figure 6A). Clinical signs of arthritis in the CIA mice treated with PBS started to be observable on day 25. The incidence of CIA was more than $50 \%$ on day 31 and all animals were affected 40 days after disease induction. F. hepatica did not reduce the clinical arthritis score $(11.75 \pm 2.96$ extract vs $14.00 \pm$ 2.56 control; $p=0.126$ ) (Figure 6B). However, it changed the time-course development of the disease since mice treated with $F$. hepatica extract presented a later beginning of clinical signs of arthritis that started to be observable only at day 31 , with more than $50 \%$ of mice affected only by day 36 . Only on day 43 all mice presented clinical signs of arthritis (Figure 6C). On the final day of the experimental period (day 46 after $\mathrm{CI}$ induction), mice treated with $F$. hepatica extract had a lower mean clinical score in the hind limbs ( $2.94 \pm 0.82$ extract vs $3.62 \pm 0.52$ control; $p=0.065$ ) (Figure 6D). Additionally, the treatment group weighed more at the end of the experimental period $(1.68 \% \pm 2.32$ extract vs $-1.76 \% \pm 3.83$ control; $p<0.05$ ) (Figure 6E/ Figure 6F).

\section{Discussion}

Despite the treatments available to induce and maintain RA remission, there is no cure. In addition to causing side effects and significant health care costs, conventional treatments are not effective for all patients. In vitro culture and animal models are very useful for assessing new RA therapies, allowing control of genetic and environmental factors and testing of several doses at different time points. In this study, we evaluated F. Hepatica extract as a new strategy for RA treatment and found that it decreased FLS cell viability, adherence, migratory potential, and cell invasiveness in vitro. It reduced leukocyte migration and nociception in AIA and delayed the clinical onset of CIA, while preventing weight loss.

FLS cells are involved in the initiation and perpetuation of RA, as well as in the joint degradation process 4,23 . Despite the wealth of knowledge gained in recent years, there are still many open questions that must be answered to fully understand the role of FLS in the development of destructive RA. It is not clear how FLS become phenotypically altered and activated in RA or at which stage of the disease this occurs ${ }^{4}$. Therefore, strategies targeting these cells could be pursued to further improve outcomes in RA ${ }^{6}$.

We found decreased in the viability of FLS treated with F. hepatica extract, which indicates that the extract could affect the synovial cell layer. As a consequence of persistent local inflammation, the thickness of the synovial membrane increases to 10-15 cell layers' which mainly consist of FLS ${ }^{22}$. Sharaf at al. ${ }^{19}$ demonstrated that products excretory-secretory (ESPs) from F. hepatica had significant 
suppressive effects on lymphoproliferation, up to $74 \%$. Reducing FLS viability could decrease the progression of RA.

Besides increased viability, RA FLS have increased resistance to apoptosis, which contributes to an accumulation of cells in the rheumatoid lesion. Despite the observed effect of $F$. hepatica extract on cell viability, there was no notable effect on apoptosis and cell death according to morphometric parameters and cell surface markers. One mechanism for this resistance could be the antiapoptotic factors in synovial fluid ${ }^{24}$. Impaired apoptosis contributes to the development of the microenvironment and maintaining an inflammatory response that characterizes established RA ${ }^{22,25}$. Moreover, altering the profile of the cells involved in RA is a delicate process since it can modify/eliminate other cells that are also sensitive to the drug. Hence, the selective elimination of a subpopulation of cells such as FLS may affect other synovial cells or lead to other diseases ${ }^{26}$.

FLS has a key role in pannus formation and joint destruction ${ }^{27}$ due to the ability to adhere, invade and degrade cartilage and bone. Accordingly, our findings demonstrated that $F$. hepatica extract modifies parameters associated with the aggressive profile of FLS such as adherence, migration, and invasion potential. Adherence and mechanical forces can affect FLS via the extracellular matrix, modulating the migration and adhesion of FLS ${ }^{28}$. RA FLS adhere significantly more to collagen type IV, fibronectin, and laminin than normal FLS. Another important characteristic is the invasive profile of FLS. The in vitro FLS invasion phenotype is correlated with histological joint damage in rats ${ }^{29}$ and radiological joint destruction in RA patients ${ }^{30}$. The suppression or modification of these phenotypes may indicate a potential inhibition of the processes involved in cartilage and bone damage.

TNF is a cytokine with both pro-inflammatory and immune-regulatory properties. In our study, we observed decreased release of TNF by FLS treated with $F$ hepatica extract under inflammatory conditions, although the difference was not statistically significant, which could have been related to insufficient sample size. Clinical trials and animal studies have indicated the direct involvement of TNF in the pathogenesis of RA ${ }^{31}$. Previous study showed that $F$. hepatica can suppress pro-inflammatory cytokines 32 . Another study demonstrated this same effect in a septic shock model by administering a preparation of antigens present in the $F$. hepatica tegument ${ }^{18}$.

To corroborate our in vitro findings, we analyzed the therapeutic effect of $F$. hepatica extract in AIA and CIA, the most frequently tested animal models of arthritis in drugs that have been approved for RA treatment ${ }^{33}$. We demonstrated that $F$. hepatica extract was able to reduce leukocyte chemotaxis and acute nociception in the AIA model. Acute AIA is characterized by joint inflammation $24 \mathrm{~h}$ after induction, including swelling, leukocyte infiltration, and pronounced hyperalgesia. In RA patients, leukocyte migration is an important pathological feature of the disease, being a marker of local inflammatory activity and pain induction ${ }^{34}$. Different mechanisms are highlighted as fundamental in this process, such as autoantigen presentation, cytokine signaling, and leukocyte activation ${ }^{23}$. F. hepatica produces molecules that can modulate these mechanisms, such as the antigens present in its tegument, which 
reduced IFN- $\gamma$ and TNF- $a$ levels in mice stimulated with lipopolysaccharide ${ }^{18,35}$ as well as the cathelicidin-like helminth defense molecule 1 (FhHDM-1), which impairs macrophage antigen processing and presentation by inhibiting lysosomal acidification ${ }^{36,37}$.

In addition to pain, the initial process of leukocyte chemotaxis leads to hypercellularity, edema, and, with time, the establishment of an inflammatory environment in the joint that contributes to subsequent synovial fibroblast activation, pannus formation, and joint destruction ${ }^{34}$. All these human features of the disease are also present in the $\mathrm{CIA} \mathrm{model}^{38}$ which can be divided into three developmental stages: the induction phase, the pre-arthritis phase with the presence of autoimmunity in the absence of clinical symptoms, and the established arthritis phase ${ }^{39}$. We found that treatment with $F$. hepatica extract delayed disease onset by delaying the beginning of clinical signs for 6 days thus prolonging the prearthritis phase of the disease. Moreover, although it did not significantly diminish the global clinical score of arthritis, it reduced the clinical score in the hind paws and reduced body weight loss. These data indicate that $F$. hepatica extract as an adjuvant treatment with a DMARD can lead to an additive effect in improving clinical symptoms in immune-mediated arthritis. Interestingly, Carranza and collaborators demonstrated that $F$. hepatica extract induces a tolerogenic phenotype in dendritic cells, and when these cells were injected in mice with CIA they were able to reduce the symptom incidence and severity ${ }^{40}$.

As previously mentioned, since $F$. hepatica extract has many different molecules with potential pharmacological effects, such as cystatins, glycoproteins, and antioxidant proteins ${ }^{19,41-43}$, detailed data on the concentrations of these components is unknown and it is not possible to determine which one exerts a specific effect on the studied parameters. Hence, further studies are necessary to clarify the exact concentration of each factor. Moreover, F. hepatica extract consists mainly of proteins, and it may induce the production of neutralizing antibodies that can interfere in its efficacy over time ${ }^{44}$.

To our knowledge, this is the first study to evaluate the therapeutic effect of $F$. hepatica extract on the FLS of RA patients and in animal models of arthritis. Treatment with $F$. hepatica extract affected the viability of FLS, but did not induce apoptosis or necrosis. However, the extract reduced FLS adherence, migration potential, and invasion capacity. In addition, it showed a tendency to decrease TNF release by FLS, which indicates an immunomodulatory effect in FLS. In vivo, treatment with $F$. hepatica extract reduced the initial events of arthritis, such as leukocyte migration and acute nociception. Moreover, it showed immunomodulatory ability by delaying the appearance of arthritis while preventing body weight loss. Therefore, the characterization of promising new immunomodulatory molecules found in helminthreleased products should be pursued, as they can promote the development of new therapies. Our results indicate that $F$. hepatica extract is a potential therapy for RA due to its ability to reduce the aggressive and invasive profile of FLS, reduce leukocyte chemotaxis, and extend the time for the clinical onset of arthritis in mice models.

\section{Declarations}


The authors acknowledge the Coordenação de Aperfeiçoamento de Pessoal de Nível Superior (CAPES), the Conselho Nacional de Desenvolvimento Científico e Tecnológico (CNPq) and the Fundação de Incentivo à Pesquisa e Eventos do Hospital de Clínicas de Porto Alegre (FIPE-HCPA) for providing funding.

\section{Author Contributions}

FCG, MF and RMX designed the experiments. ECFP, FCG, JMSS, MF, MPC, RTP, MLG, SPD and THR performed the experiments. ECFP, FCG, MF, RCES, RTP and SPD analyzed the data. FCG, MF and SPD wrote the manuscript. JMSS, RCES, RMX, RTP and MLG reviewed the manuscript. AHP, HBF and RMX contributed to the discussion. All authors read and approved the manuscript.

\section{Competing Interests}

The authors declare no competing interests.

\section{References}

1. 1. Cruvinel, W. de M. et al. Células T regulatórias naturais (TREGS) em doenças reumáticas. Rev. Bras. Reumatol. 48, 342-355 (2008).

2. 2. Gibofsky A. Epidemiology, Pathophysiology, and Diagnosis of Rheumatoid Arthritis: A Synopsis. Am J Manag Care 20, S128-35 (2014).

3. 3. Pap, T., Müller-Ladner, U., Gay, R. E. \& Gay, S. Fibroblast biology. Role of synovial fibroblasts in the pathogenesis of rheumatoid arthritis. Arthritis Res. 2, 361-367 (2000).

4. 4. Ospelt, C. Synovial fibroblasts in 2017. 1-10 (2017). doi:10.1136/rmdopen-2017-000471

5. 5. Rheumatoid arthritis in adults: management. National Guideline Centre (UK) (National Institute for Health and Care Excellence (UK);, 2018). doi:10.7326/0003-4819-66-1-246_3

6. 6. Tanaka, Y. Current concepts in the management of rheumatoid arthritis. Korean J. Intern. Med. 31, 210-218 (2016).

7. 7. Luqmani, R. \& Cox, M. DMARDs. National Rheumatoid Arthritis Society (2016).

8. 8. Matisz, C. E., McDougall, J. J., Sharkey, K. A. \& McKay, D. M. Helminth parasites and the modulation of joint inflammation. J. Parasitol. Res. 2011, (2011).

9. 9. Langdon, K. et al. Helminth-based therapies for rheumatoid arthritis: A systematic review and meta-analysis. Int. Immunopharmacol. 66, 366-372 (2019).

10. 10. Gomides APM, Ima CMBLL, Rocha FAC, Henrique da Mota LM, M. de O. S. Parasites in Rheumatoid Arthritis: Imminent Threat or Protective Effect? Curr Rheumatol Rev 13, 80-85 (2017).

11. 11. Sipahi, A. M. \& Baptista, D. M. Helminths as an alternative therapy for intestinal diseases. World J. Gastroenterol. 23, 6009-6015 (2017).

12. 12. Correale, J. \& Farez, M. Association between parasite infection and immune responses in multiple sclerosis. Ann. Neurol. 61, 97-108 (2007). 
13. 13. Summers, R. W., Elliott, D. E., Urban, J. F., Thompson, R. A. \& Weinstock, J. V. Trichuris suis therapy for active ulcerative colitis: A randomized controlled trial. Gastroenterology 128, 825-832 (2005).

14. 14. Maruszewska-Cheruiyot, M., Donskow-Lysoniewska, K. \& Doligalska, M. Helminth therapy: Advances in the use of parasitic worms against Inflammatory Bowel Diseases and its challenges. Helminthol. 55, 1-11 (2018).

15. 15. Saunders, K. A., Raine, T., Cooke, A. \& Lawrence, C. E. Inhibition of autoimmune type 1 diabetes by gastrointestinal helminth infection. Infect. Immun. 75, 397-407 (2007).

16. 16 . Helmby, H. Human helminth therapy to treat inflammatory disorders- where do we stand? $B M C$ Immunol. 16, 1-5 (2015).

17. 17. Abath, F. G. C. \& Werkhauser, R. C. The tegument of Schistosoma mansoni: Functional and immunological features. Parasite Immunol. 18, 15-20 (1996).

18. 18. Hamilton, C. M. et al. The Fasciola hepatica tegumental antigen suppresses dendritic cell maturation and function. Infect. Immun. 77, 2488-2498 (2009).

19. 19. Sharaf, O. F., Amir, E. M. \& Hawash, Y. A. LYMPHO-PROLIFERATIVE RESPONSES TO VARIOUS FASCIOLA HEPATICA WORM'S ANTIGENS: AN IN VITRO STUDY. J Egypt Soc Parasitol 46, (2016).

20. 20. Khaznadji, E., Collins, P., Dalton, J. P., Bigot, Y. \& Moiré, N. A new multi-domain member of the cystatin superfamily expressed by Fasciola hepatica. Int. J. Parasitol. 35, 1115-1125 (2005).

21. 21. Wilson, R. A. et al. Exploring the Fasciola hepatica tegument proteome. Int. J. Parasitol. 41, 1347-1359 (2011).

22. 22. Bartok, B. \& Firestein, G. S. Fibroblast-like synoviocytes: Key effector cells in rheumatoid arthritis. Immunol. Rev. 233, 233-255 (2010).

23. 23. Smolen, J. S., Aletaha, D. \& Mclnnes, I. B. Rheumatoid arthritis. Lancet 388, 2023-2038 (2016).

24. 24. Dennis, G. et al. Synovial phenotypes in rheumatoid arthritis correlate with response to biologic therapeutics. Arthritis Res. Ther. 16, (2014).

25. 25. Manferdini, C. et al. From osteoarthritic synovium to synovialderived cells characterization: Synovial macrophages are key effector cells. Arthritis Res. Ther. 18, 1-13 (2016).

26. 26. Lumb, F. E. et al. Dendritic cells provide a therapeutic target for synthetic small molecule analogues of the parasitic worm product, ES-62. Sci. Rep. 7, 1-13 (2017).

27. 27. Doonan, J. et al. Protection against arthritis by the parasitic worm product ES-62, and its drug-like small molecule analogues, is associated with inhibition of osteoclastogenesis. Front. Immunol. 9, (2018).

28. 28. Casnici, C. et al. Optimized 'In Vitro' Culture Conditions for Human Rheumatoid Arthritis Synovial Fibroblasts. Mediators Inflamm. 2014, (2014).

29. 29. Korb, A., Pavenstädt, H. \& Pap, T. Cell death in rheumatoid arthritis. Apoptosis 14, 447-454 (2009).

30. 30. Childs, B. G., Baker, D. J., Kirkland, J. L., Campisi, J. \& Deursen, J. M. Senescence and apoptosis: dueling or complementary cell fates? EMBO Rep. 15, 1139-1153 (2014). 
31. 31. Lubberts, E. \& Berg, W. B. van den. Cytokines in the Pathogenesis of Rheumatoid Arthritis and Collagen-Induced Arthritis. Adv Exp Med Biol 520, 194-202 (2003).

32. 32. Gabriel, S. E. The epidemiology of rheumatoid arthritis. Rheum. Dis. Clin. North Am. 27, 269-281 (2001).

33. 33. Hegen, M., Keith, J. C., Collins, M. \& Nickerson-Nutter, C. L. Utility of animal models for identification of potential therapeutics for rheumatoid arthritis. Ann. Rheum. Dis. 67, 1505-1515 (2008).

34. 34. Mclnnes, I. B. \& Schett, G. The pathogenesis of rheumatoid arthritis. N. Engl. J. Med. 365, 220519 (2011).

35. 35. Vukman, K. V., Adams, P. N., Metz, M., Maurer, M. \& O’Neill, S. M. Fasciola hepatica Tegumental Coat Impairs Mast Cells' Ability To Drive Th1 Immune Responses. J. Immunol. 190, 2873-2879 (2013).

36. 36. Liu, Q. et al. Proteomic analysis of Fasciola hepatica excretory and secretory products (FhESPs) involved in interacting with host PBMCs and cytokines by shotgun LC-MS/MS. Parasitol. Res. 116, 627-635 (2017).

37. 37. Robinson, M. W. et al. A helminth cathelicidin-like protein suppresses antigen processing and presentation in macrophages via inhibition of lysosomal vATPase. FASEB J. 26, 4614-4627 (2012).

38. 38. Brand, D. D., Latham, K. A. \& Rosloniec, E. F. Collagen-induced arthritis. Nat. Protoc. 2, $1269-75$ (2007).

39. 39. Dekkers, J. S., Schoones, J. W., Huizinga, T. W., Toes, R. E. \& Van Der Helm-Van Mil, A. H. Possibilities for preventive treatment in rheumatoid arthritis? Lessons from experimental animal models of arthritis: A systematic literature review and meta-analysis. Ann. Rheum. Dis. 76, 458-467 (2017).

40. 40. Carranza, F. et al. Helminth antigens enable CpG-activated dendritic cells to inhibit the symptoms of collagen-induced arthritis through Foxp3+ regulatory T cells. PLoS One 7, (2012).

41. 41. Cancela, M. et al. Functional characterization of single-domain cystatin-like cysteine proteinase inhibitors expressed by the trematode Fasciola hepatica. Parasitology 144, 1695-1707 (2017).

42. 42. Ravida, A. et al. Fasciola hepatica surface tegument: Glycoproteins at the interface of parasite and host. Mol. Cell. Proteomics 15, 3139-3153 (2016).

43. 43. Dalton, J. P., Robinson, M. W., Mulcahy, G., O’Neill, S. M. \& Donnelly, S. Immunomodulatory molecules of Fasciola hepatica: Candidates for both vaccine and immunotherapeutic development. Vet. Parasitol. 195, 272-285 (2013).

44. 44. Smith, A. et al. Unraveling the Effect of Immunogenicity on the PK/PD, Efficacy, and Safety of Therapeutic Proteins. J. Immunol. Res. 2016, (2016).

45. 45. Aletaha, D. et al. 2010 Rheumatoid arthritis classification criteria: an American College of Rheumatology/European League Against Rheumatism collaborative initiative. Arthritis Rheum. 62, 2569-81 (2010). 
46. 46. Cancela, M. et al. A distinctive repertoire of cathepsins is expressed by juvenile invasive Fasciola hepatica. Biochimie 90, 1461-1475 (2008).

47. 47. Grespan, R. et al. CXCR2-specific chemokines mediate leukotriene B4-dependent recruitment of neutrophils to inflamed joints in mice with antigen-induced arthritis. Arthritis Rheum. 58, 2030-2040 (2008).

\section{Figures}

(A)

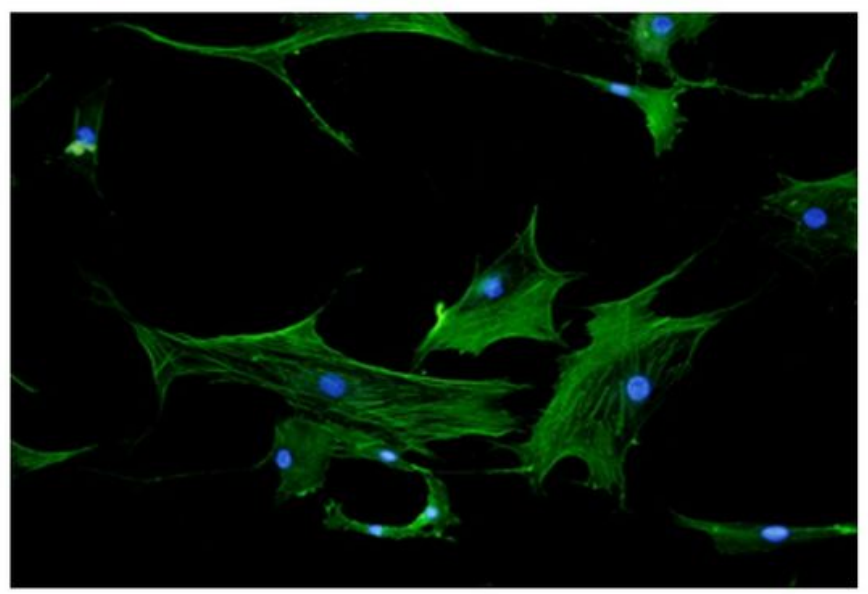

(C)
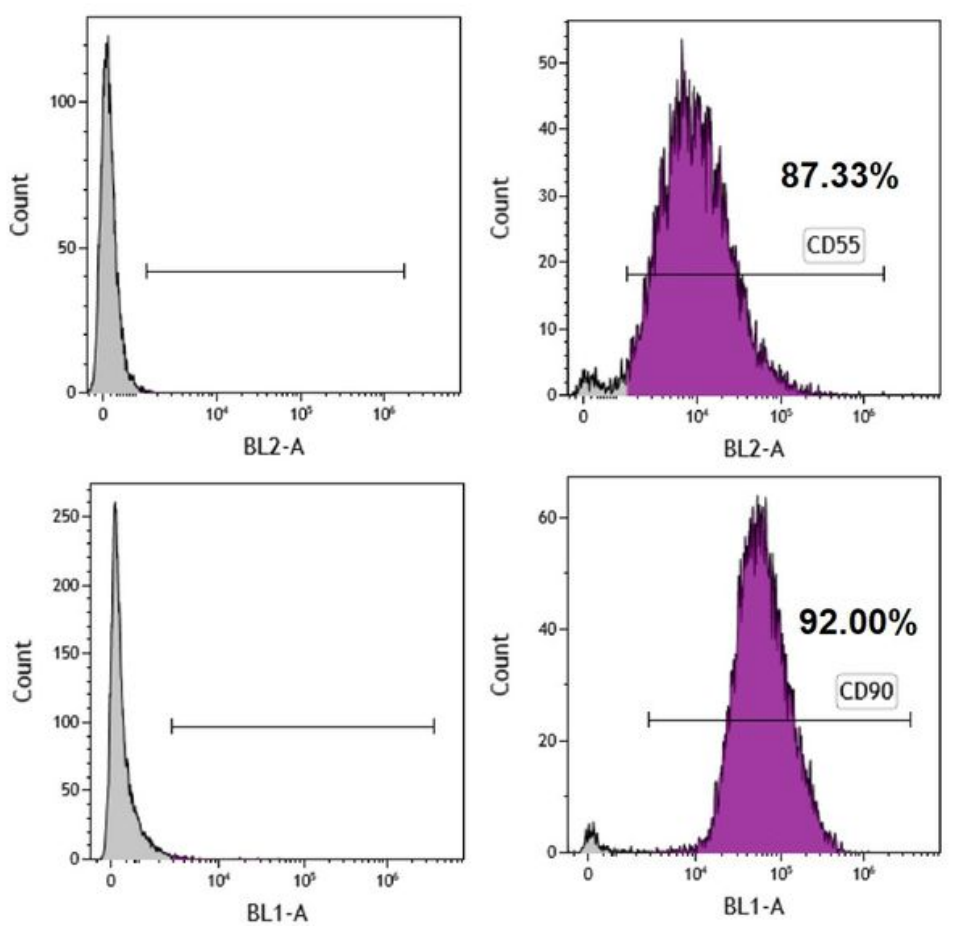

(B)

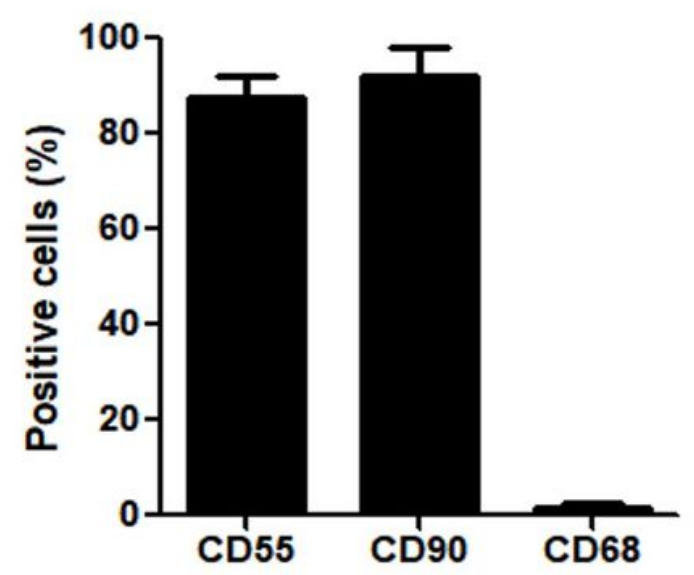

Figure 1

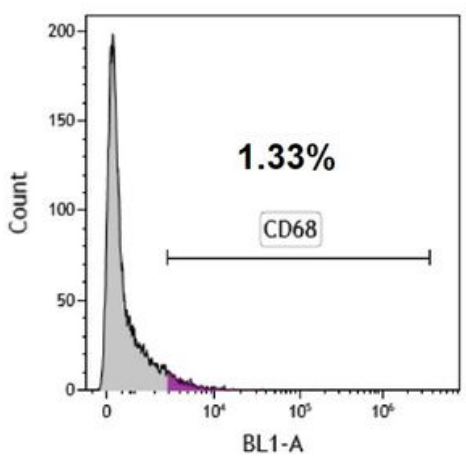


Characterization of FLS cultures. (A) Representative image of FLS morphology labeled with Phalloidin for actin filamentous, F-actin (green), and DAPI for nuclei (blue). (B) Percentage of positive cells for CD55, CD90 (both FLS markers), and CD68 (macrophage marker). (C) Representative of flow cytometry analysis for CD55, CD90, and CD68. Data are expressed as mean \pm SD.

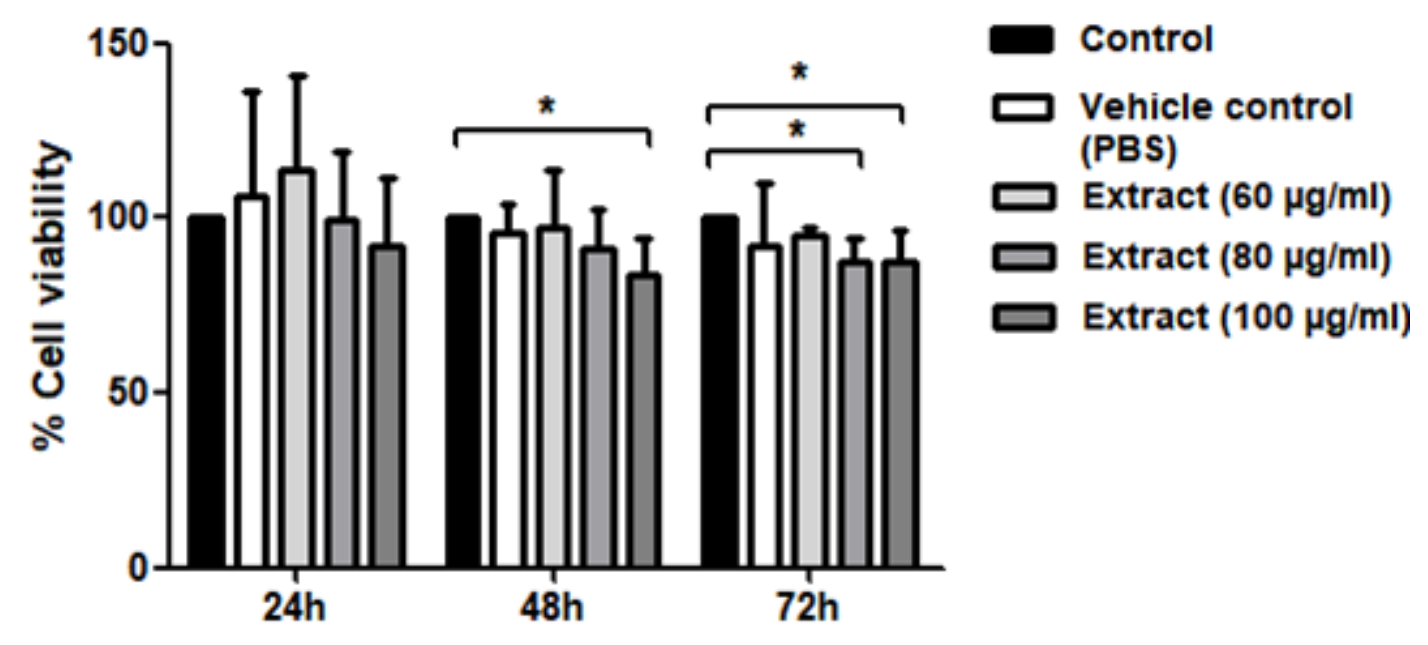

Figure 2

F. hepatica extract decreased the FLS viability. F. hepatica extract was tested at different concentration (0, $60,80,100 \mathrm{mg} / \mathrm{mL})$ and timepoints $(24,48$ and $72 \mathrm{~h}$ ) in FLS cultures. Cell viability was evaluated by MTT test. A decrease in cell viability was observed at concentration of $100 \mu \mathrm{g} / \mathrm{mL}$ after $48 \mathrm{~h}$ and at concentrations of $80 \mu \mathrm{g} / \mathrm{mL}$ and $100 \mu \mathrm{g} / \mathrm{mL}$ after $72 \mathrm{~h}$, when compared with the control group without treatment. ${ }^{*} p<0.05$; ANOVA two-way. $(\mathrm{n}=8)$. Data were normalized as percent of control at each time point and is expressed as mean $\pm S D$. 
(A

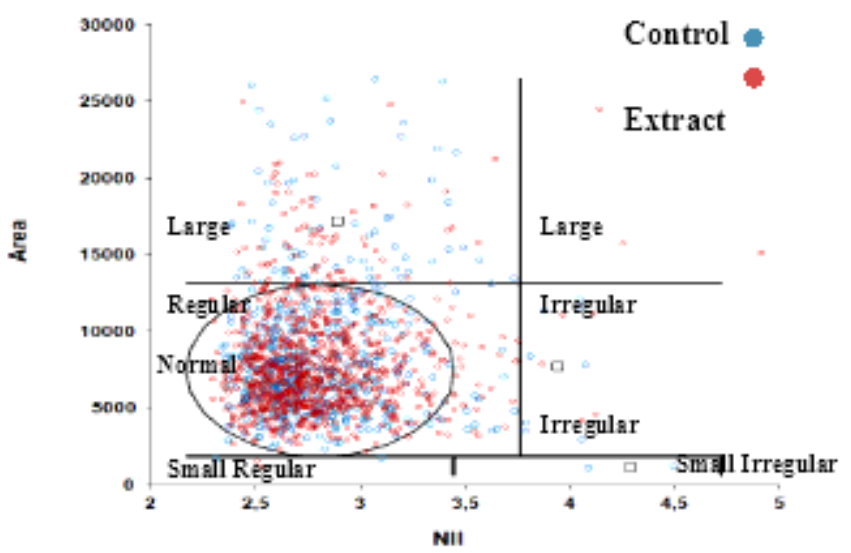

(C)
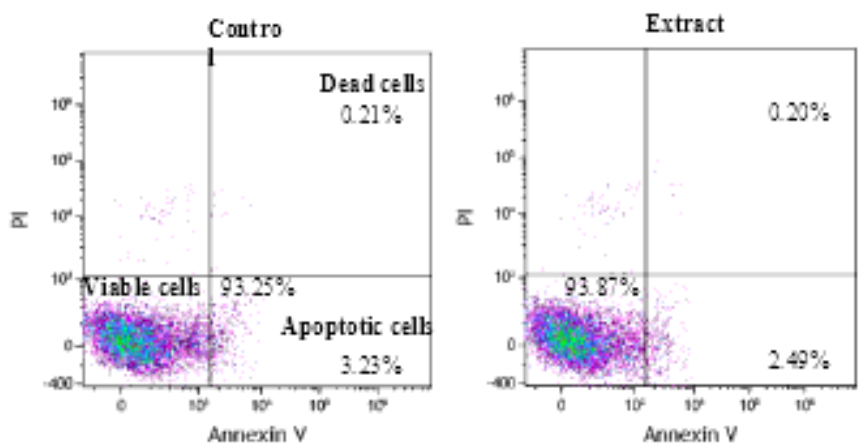

(B)

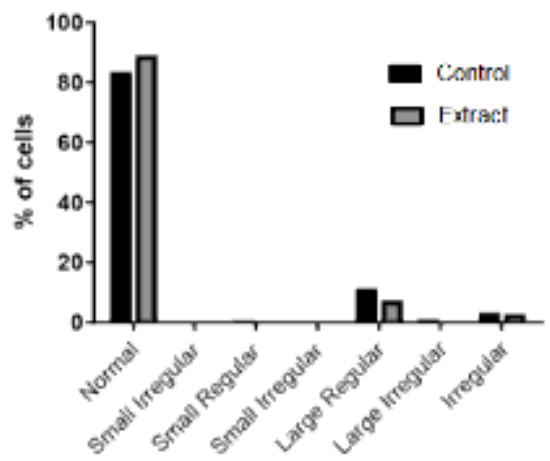

(D)

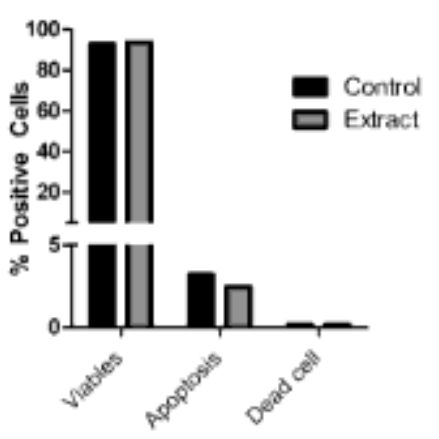

Figure 3

F. hepatica extract did not alter parameters of nuclear morphometry, apoptosis, cell death of FLS. (A) Nuclear morphometric analysis was perfomed in FLS cell after $48 \mathrm{~h}$ culture with $F$. hepatica extract (100 $\mathrm{mg} / \mathrm{mL}$ ). Representative analysis in parameters of area, roundness, aspect, radius ratio, area box, and the nuclear irregularity index NII in parameters of nuclear irregularity when compared with the untreated group. Red dots: control; blue dots: F. hepatica extract; $(n=3)(B)$ Percentage of positive cells for viability parameters $(n=3)$. (C) Representative flow cytometry analysis of apoptosis (Annexin $V$ ) and necrosis (propidium iodide); Control (left) and treated (right). (D) Percentage of viable, apoptotic, and dead cells $(n=4)$. Data are expressed as mean \pm SD. 
(A)

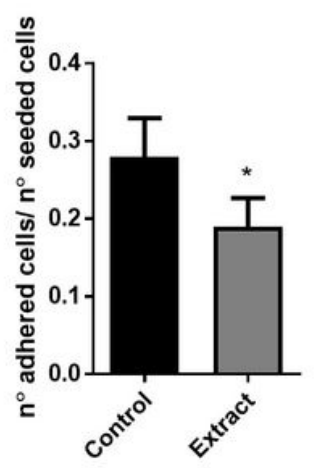

(D)

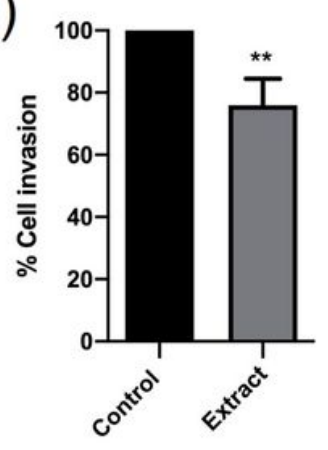

(B)

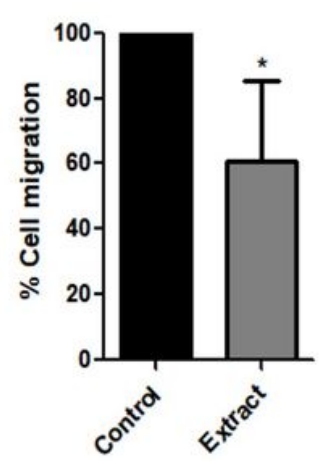

(E)
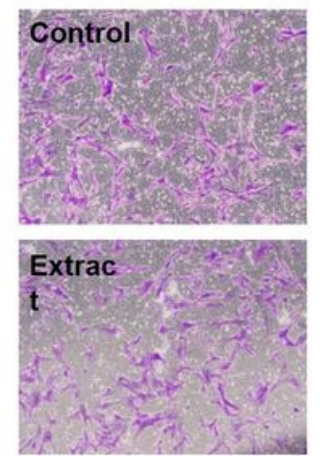

(C)
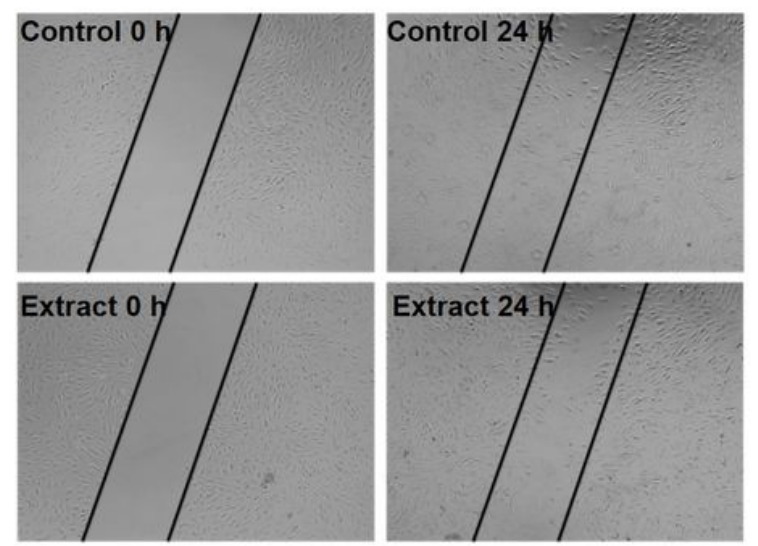

(F)

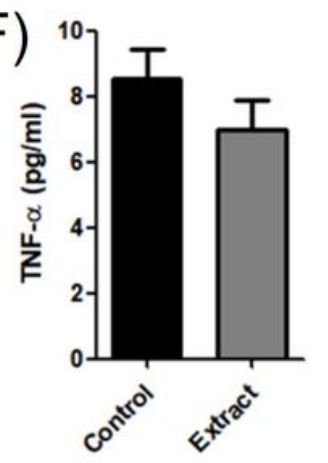

Figure 4

F. hepatica extract reduced the adherence efficiency, the migratory, and invasion potentials of FLS. (A) Adherence capacity of FLS was analyzed after $6 \mathrm{~h}$ of $F$. hepatica extract incubation $(100 \mathrm{mg} / \mathrm{mL})(\mathrm{n}=4)$. (B) Migratory potential of FLS after wound healing $(n=3)$. (C) Representative microscopy images of FLS migration assay. (D) Invasive capacity of FLS was evaluated by matrigel assay $(n=3)$. (E) Representative images of stained cells that invaded through matrigel invasion chambers. (F) TNF-a secretion by FLS after INF-g stimulation $(n=3)$. Original magnification: $20 x$. T Test; Data are expressed as mean $\pm S D ; p<$ 0.05 . 
(A)

(B)

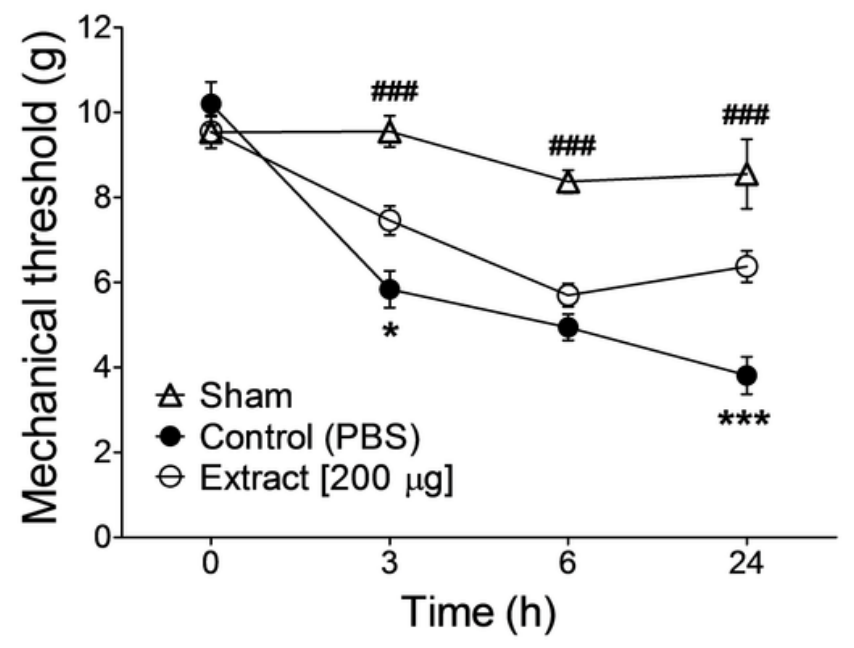

(C)

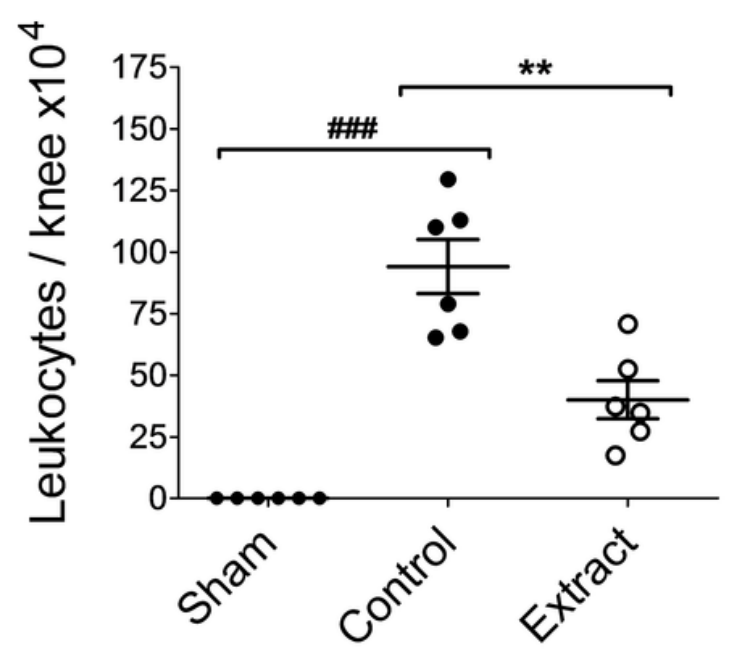

Figure 5

F. hepatica extract decreased nociception and leukocytes chemotaxis in AIA. (A) Illustration of experimental design (B) Nociception was determined at 0, 3, 6 and $24 \mathrm{~h}$ after mBSA injection (C) Counting of leukocytes in the knee of mice $24 \mathrm{~h}$ after injection with mBSA. ANOVA followed by Bonferroni post hoc

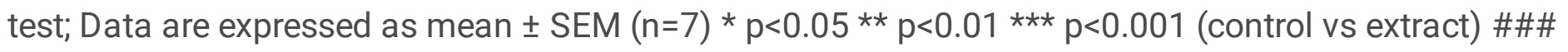
$\mathrm{p}<0.001$ (control vs sham) 
(A)

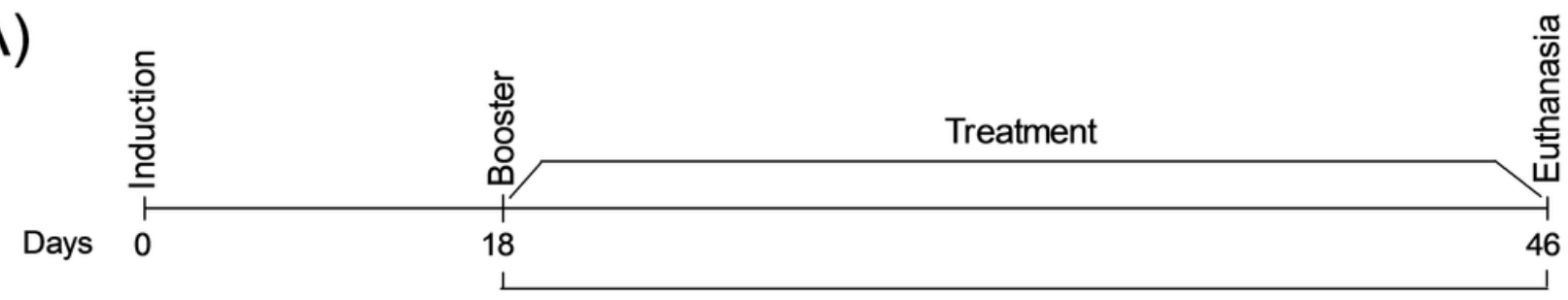

Evaluation of clinical arthritis score, incidence of arthriris and body weight

(B)

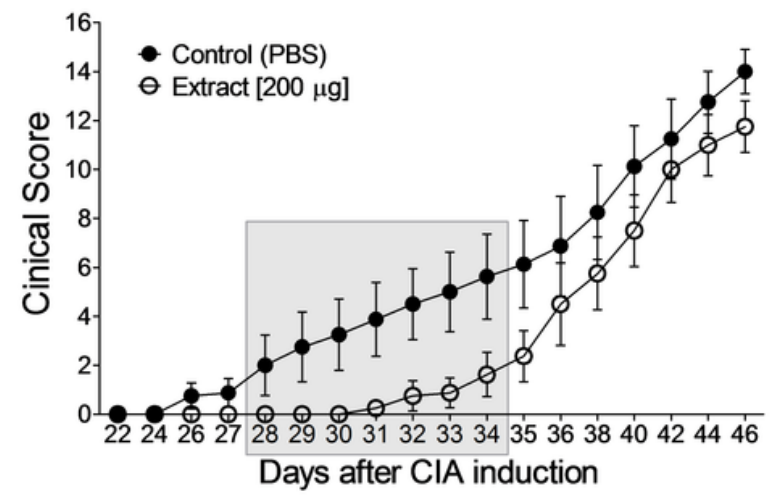

(D)

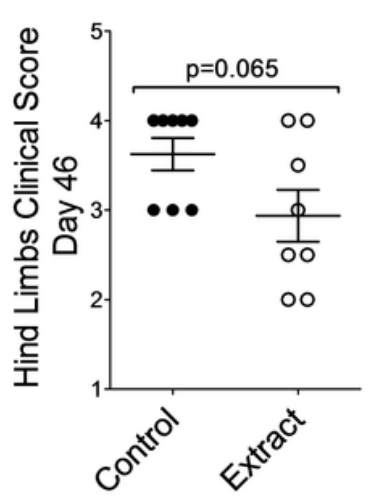

(C)

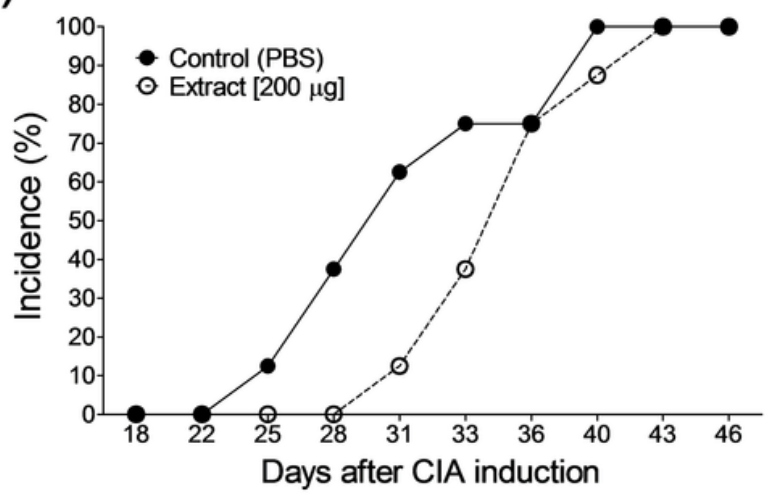

$(\mathrm{F})$

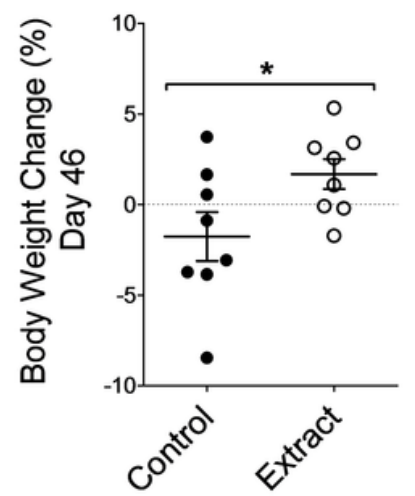

Figure 6

F. hepatica delayed the beginning of clinical signs of arthritis and prevented body weight loss in CIA. (A) Illustration of experimental design (B) Clinical score of arthritis during CIA development (C) Incidence of arthritis (D) Clinical score of arthritis in the hind limbs at the last day of the CIA experiment (E) Body weight change $(F)$ Body weight change at the last day of experimentation. Data are expressed as mean \pm SEM ( $n=8)$. (B,C,E) Two-way ANOVA followed by Bonferroni post hoc test; (D, F) Student's T-test. ** $\mathrm{p}<0.01$.

\section{Supplementary Files}


This is a list of supplementary files associated with this preprint. Click to download.

- Supplementaryinformation.docx 